\title{
DIMETHYL ETHER AS A DRIFT-CHAMBER GAS
}

\author{
G. BARI, M. BASILE, G. BONVICINI, G. CARA ROMEO, R. CASACCIA, L. CIFARELLI, \\ F. CINDOLO, A. CONTIN, G. D'ALI, C. DEL PAPA, S. FOCARDI, G. IACOBUCCI, \\ G. MACCARRONE, T. MASSAM, F. MOTTA, R. NANIA, F. PALMONARI, G. PRISCO, \\ G. SARTORELLI, G. SUSINNO, L. VOTANO and A. ZICHICHI \\ Dipartimento di Fisica dell'Università, Bologna, Italy \\ Istituto Nazionale di Fisica Nucleare, Bologna, Italy \\ CERN, Geneva, Switzerland \\ Istituto Nazionale di Fisica Nucleare, LNF, Frascati, Italy \\ University of Michigan, Ann Arbor, Mich., USA \\ Dipartimento di Fisica, Università di Palermo, Italy
}

Received 22 May 1986

\begin{abstract}
We have continued the testing of dimethyl ether as a drift-chamber gas in order to improve the understanding of its properties. In particular, we report on measurement accuracy, on systematic effects, and some preliminary data on the ageing of a detector filled with dimethyl ether.
\end{abstract}

\section{Introduction}

After the positive results of preliminary studies on the use of dimethyl ether (DME) in time expansion chambers (TECs) [1,2], some questions remained to be answered in order to be confident about the use of DME in actual experiments. First, the gain gap is a non-negligible part $(\sim 10 \%)$ of the active volume of the chamber and it would be unacceptable if tracks going through it could not be reconstructed or the spatial resolution were excessively deteriorated. Using a simply calculated time-to-distance relation (TDR), we show that this is not the case; in addition, it has become apparent to us that the drift-gap field can be extended towards the wire, past the mesh, further improving the resolution in the gain gap. Secondly, it was important to show that the expected track distortions due to a not perfectly constant drift field can be dealt with: we demonstrate that, at least for our particular chamber, the distortions can be almost totally eliminated.

Finally, we dispel the fear of premature ageing of the sense wires of a chamber using DME.

\section{Accuracy}

The test chamber for accuracy measurements has been described elsewhere [2]. The only two changes were a reduction of the drift gap from 3 to $2 \mathrm{~cm}$ (fig. $1 \mathrm{a})$, and the use of thinner $(1 \mathrm{~mm})$ potential plates. This was done to improve the shape of the electric field in the drift gap (see below). The diameter of the sense wires was $20 \mu \mathrm{m}$. We have also improved the rise time of the amplifiers preceding the discriminators (LRS $620 \mathrm{CL}$ ) from 12 to $2 \mathrm{~ns}$, in an attempt to minimize pulse-slewing effects.

A $3.5 \mathrm{GeV} / c \pi^{-}$beam illuminated the drift space (drift gap) and the space between the sense wire and the mesh (gain gap). A coincidence between scintillation counters in the beam and a small-surface $\left(2 \times 1 \mathrm{~cm}^{2}\right)$ counter close to the chamber gave the starting pulse to an LRS 4208 digital timer. Data were taken in three running conditions:

$V_{\mathrm{s}}=2.3 \mathrm{kV} \quad V_{\mathrm{f}}=1.5 \mathrm{kV} \quad p=1$ atm $E / p=4.0 \mathrm{~V} / \mathrm{cm}$ Torr,

$V_{\mathrm{s}}=2.3 \mathrm{kV} \quad V_{\mathrm{f}}=1.5 \mathrm{kV} \quad p=1 \mathrm{~atm} \quad E / p=2.6 \mathrm{~V} / \mathrm{cm}$ Torr,

$V_{\mathrm{s}}=3.83 \mathrm{kV} \quad V_{\mathrm{f}}=1.5 \mathrm{kV} \quad p=3 \mathrm{~atm} \quad E / p=1.7 \mathrm{~V} / \mathrm{cm}$ Torr,

where $V_{\mathrm{s}}$ and $V_{\mathrm{f}}$ are the sense and field wire potentials, $p$ is the pressure, and $E$ the drift field.

The electric field is (almost) constant in the drift gap (see below), but not in the gain gap. No optimization of the field inside the gain gap was attemped; however, it would have been possible to shape the field in the gain gap, in order to smoothly match the field in the drift gap and make it constant for most of the distance between the mesh and the wire plane, with a predictable improvement in spatial resolution (see our TEC vertex detector design [3]).

In order to calculate the spatial resolution, we have used the same algorithm as in ref. [2] in the drift gap; 

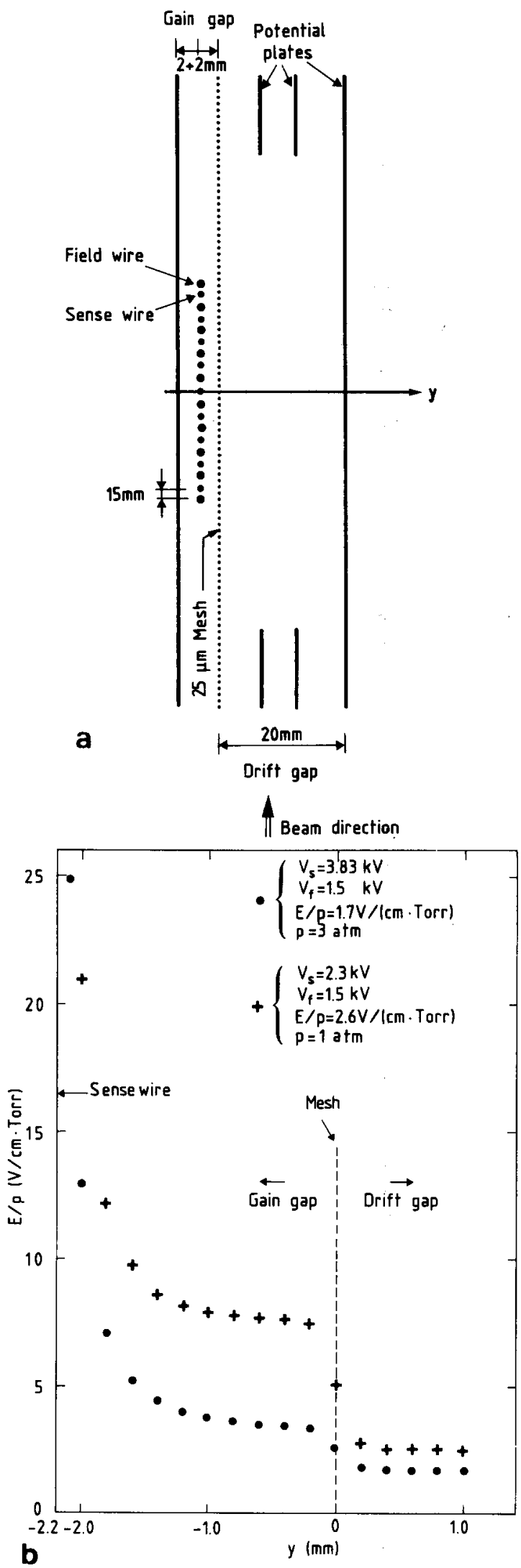

however, in the gain gap, where the TDR is nonlinear, we have modeled it using a Monte Carlo code written by J. Va'vra. A better way of deriving the TDR would have been to use a $\mathrm{N}_{2}$ laser [4]. We have taken as trigger time the mean value of the distribution of the arrival times of the first electron on the wire for 20 tracks generated in the same position. To calculate the arrival time, we needed the dependence of the drift velocity $v$ on the reduced electric field. The diffusion of the electrons has been ignored in this calculation. Data on electron drift velocity in DME as a function of $E / p$ were collected, both from our measurements and from the literature [5]; the data have been fitted with a third-order polynomial over five intervals, requiring continuity of the function and of its first derivative:

$v=A(E / p)^{3}+B(E / p)^{2}+C(E / p)+D$.

The velocity is in units of $\mu \mathrm{m} / \mathrm{ns}$ and $E / p$ in units of $\mathrm{V} / \mathrm{cm}$ Torr. The values of the parameters are given in table 1 (notice that no data are available for $E / p>15.4$ ). Once the TDR has been calculated, the procedure to obtain the resolution is the same as that used in ref. [2].

Figs. 2 and 3 show the results at 1 and 3 atm, respectively; the resolution (defined as the average resolution for all the wires) is plotted against the drift distance. The vertical dotted line represents the position of the mesh separating the gain from the drift gap. At 1 atm, near the mesh, the value of $E / p$ in the gain gap is higher by a factor of 3 than in the drift gap (fig. 1b); the subsequent increase in drift velocity produces a deterioration of the spatial resolution. At $3 \mathrm{~atm}$, the electric field transition across the mesh turns out to be almost undetectable; therefore, the resolution in the gain gap remains excellent. We thus conclude from figs. $1 \mathrm{~b}$ and 2 that, had the electric field been appropriately shaped, as explained above, the resolution in the last two bins of the gain gap for the data taken at $1 \mathrm{~atm}$ would have been $-30 \mu \mathrm{m}$. The effect of primary ionization statistics is clearly visible in the first bin; in any case, the low statistics in the gain gap $(\sim 100$ events per bin) did not allow a detailed understanding of the behaviour of the resolution close to the wire, and the error bars are mostly due to fluctuations in the resolution measured from wire to wire. In addition, in the gain gap it is impossible to resolve the left-right ambiguity, and this must have worsened the measured resolu-

Fig. 1. (a) Cell geometry, showing drift gap, gain gap, potential plates, field and sense wires, mesh. (b) Value of the component of the electric field along the drift direction as a function of distance from the wires. At 3 atm there is a much smaller discontinuity at the mesh than at $1 \mathrm{~atm}$. We attribute to this fact the smaller discontinuity of the spatial resolution in the data at $3 \mathrm{~atm}$ in crossing the mesh (figs. 2,3 ). 
Table 1

Values of the parameters of the fit to the drift velocity

\begin{tabular}{llllr}
\hline $\begin{array}{l}E / P \text { range } \\
\text { [V/cm Torr] }\end{array}$ & $A$ & $B$ & $C$ & \multicolumn{1}{l}{$D$} \\
\hline $0.5-1.35$ & 0.106 & 0.056 & 1.718 & 0.295 \\
$1.35-3.4$ & 0.0500 & 0.0845 & 1.966 & 0.055 \\
$3.4-7.1$ & 0.02098 & 0.00229 & 3.138 & -2.285 \\
$7.1-15.4$ & -0.01127 & 0.00234 & 8.015 & -25.369 \\
15.4 & 0 & 0 & 0 & 57.100 \\
\hline
\end{tabular}

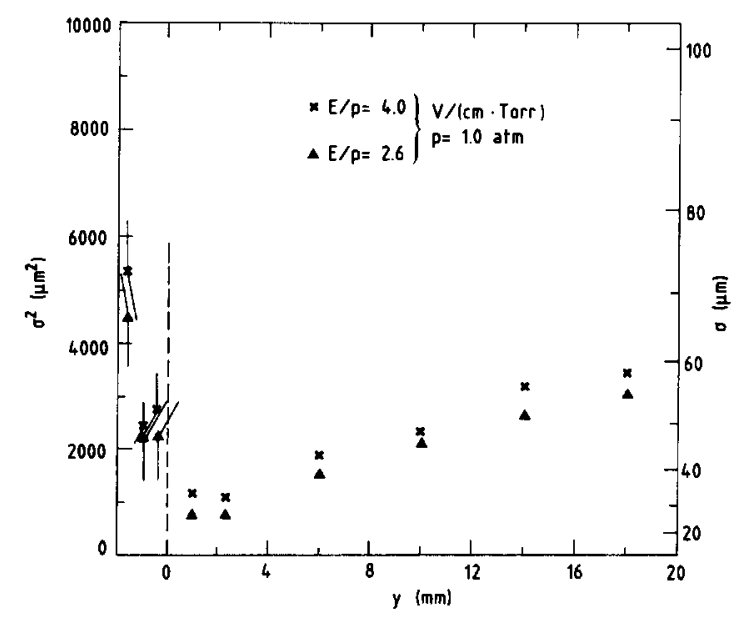

Fig. 2. Spatial resolution in the drift as well as in the gain gap at $1 \mathrm{~atm}$. Error bars refer to $\sigma^{2}$.

tion. The data in figs. 2 and 3 show that the increase in bandwidth of the amplifiers did not affect the accuracy; in fact, the values reported are similar to those of ref. [2].

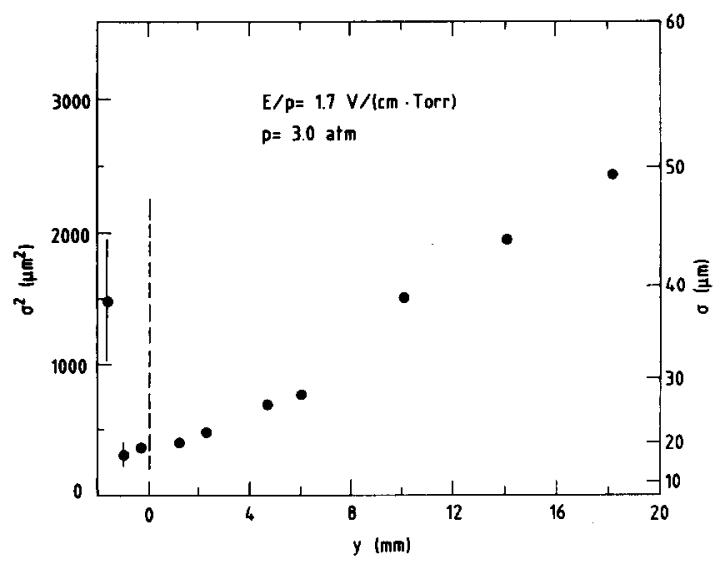

Fig. 3. Spatial resolution in the drift as well as in the gain gap at $3 \mathrm{~atm}$. Error bars refer to $\sigma^{2}$.

\section{Systematic effects}

The electron drift velocity in DME depends upon the electric field almost linearly in the range of $E / p$ values where we have worked. Therefore the conversion from drift-time to position requires either a very uniform field, or a precise knowledge of it. In our geometry (fig. 1a), the electric field in the drift gap has two boundaries; a metallic plate and a $25 \mu \mathrm{m}$ mesh $(80 \%$ transparent); two electrodes at intermediate potential eliminate edge effects. Extending the wire plane would have reduced the sagging of the equipotentials.

The study of track deformation has gone through various steps. The points have been fitted with straight lines to bin the tracks in $y$ and calculate their angle relative to the $y$ axis. A set of (undistorted) tracks close to the mesh $(0.2 \mathrm{~mm} \leqslant y \leqslant 2 \mathrm{~mm})$ have been used to correct for different cable lengths, wire displacements, etc., using the usual iterative procedure of centring to zero the distributions of residuals, which did not, initially, show any systematics in that bin. We would like to stress that, using tracks from all $y$ bins, the procedure does not converge (i.e. there are systematic distortions for all tracks).

Having thus corrected all points for the individual zeros of time, we have done a second fit, using parabo-

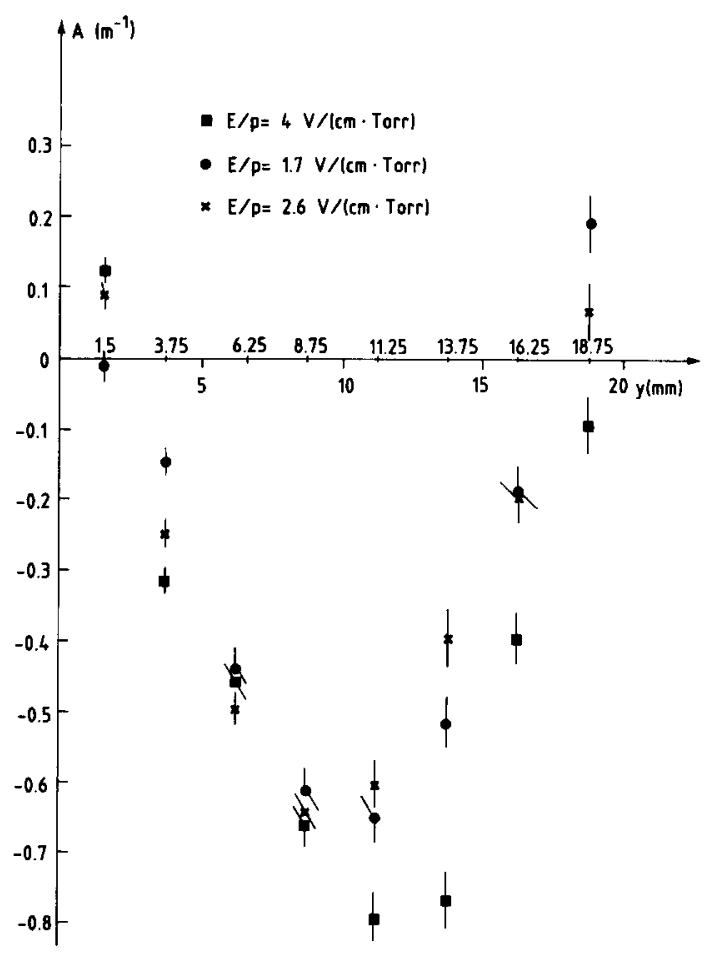

Fig. 4. Values of the quadratic coefficient for three different running conditions. 
las whose vertices correspond to the central wire position, for clear reasons of symmetry, and whose axes are rotated to be parallel to the drift direction. The values of the quadratic coefficient $A=A(y)$ of the fit (whose geometrical meaning is the inverse of the radius of curvature) show the same dependence on the drift distance (fig. 4) for the three running conditions specified above. The maximum sagitta of the track is $\leqslant 30 \mu \mathrm{m}$, corresponding to a minimum apparent momentum $\geqslant 1$ $\mathrm{GeV} / c$ in a field of $2 \mathrm{~T}$, the track length being $18 \mathrm{~mm}$. Knowing the average curvature of the tracks as a function of $y$, we simply add to the $y$ coordinate of each point a length which is calculated according to this curvature. After this first correction, the maximum residual sagitta is $1.5 \mu \mathrm{m}$, corresponding to an apparent momentum $\sim 20 \mathrm{GeV} / c$.

We would like to stress that the above procedure does not improve the spatial resolution of each point although, of course, it is essential to the finding of secondary vertices or for measuring momenta.

\section{Ageing}

We have investigated ageing effects in DME using an $\mathrm{Al}$ tube with a concentric gold-plated tungsten wire of $20 \mu \mathrm{m}$ diameter, at a voltage of $5.5 \mathrm{kV}$. The tube was filled with DME flowing at about four changes of gas per hour. To irradiate the wire, we used a ${ }^{60} \mathrm{Co}$ source of $16 \mu \mathrm{Ci}$. The gain was about $4 \times 10^{5}$, corresponding to $-1 \mu \mathrm{A}$ of current drawn from the wire. The current did not change for the two months the test lasted. In the end, we collected more than $10 \mathrm{C}$ of total charge. Since part of the wire was shielded from the source with a 5 $\mathrm{cm}$ thick $\mathrm{Pb}$ brick, and taking the source off the tube made the current drop essentially to zero, we assume that the whole charge went through the irradiated part of the wire. During the test, we did not carefully record the pulse height; however, we did notice a drop in gain at the beginning, though it seemed to remain constant afterwards. Upon opening the tube, at the end of the test, we found that the irradiated part was clearly distinguishable from the rest of the wire, since its diameter had visibly increased (to about $60 \mu \mathrm{m}$, see fig. 5a). In addition, the length of the damaged part was $2.5 \mathrm{~cm}$ : i.e. we had collected $4 \mathrm{C} / \mathrm{cm}$.

An examination of the deposits on the wire, done under an electron microscope, showed that they were made of hard scales not easily cleaned off (fig. 5b). No whiskers were found. The deposits were insulating. X-ray fluorescence analysis showed that there were quantities of $\mathrm{Cl}, \mathrm{S}$. Si, $\mathrm{Ca}$, and probably $\mathrm{F}$. No element with $Z$ below 9 could be detected with the instrument used. A search for the origin of these impurities has yielded the following results. Firstly, we found that the Rilsan tubing used in the gas system clearly contains $S$; it was
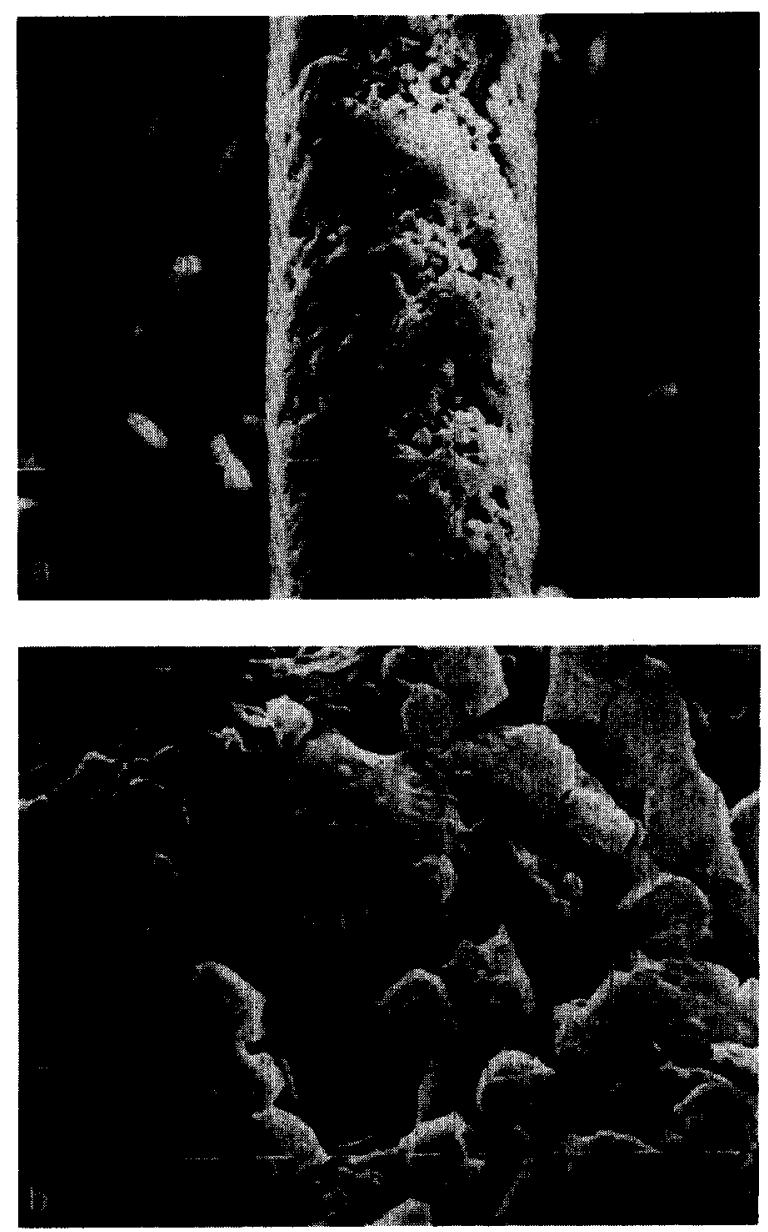

Fig. 5. (a) Photograph of the wire after being exposed to 4 $\mathrm{C} / \mathrm{cm}$. Each marker is $10 \mu \mathrm{m}$. Enlargement is 640 times. (b) Photograph of the wire after being exposed to $4 \mathrm{C} / \mathrm{cm}$. Each marker is $1 \mu \mathrm{m}$. Enlargement is 5000 times.

therefore a primary suspect as a source of this impurity. Oil, used in tapping a hole, was another possible source of $\mathrm{S}$ as well as $\mathrm{Si}$ (oil was also contained in the gas used; see table 2, Schweissen Technik). In any case $\mathrm{Si}$ is

Table 2

The contaminants as stated by various manufacturers

\begin{tabular}{lll}
\hline Manufacturer & $\begin{array}{l}\text { Purity } \\
{[\%]}\end{array}$ & Contaminant \\
\hline Fluka & 99.2 & $0.8 \%$ Alkane, \\
& & $0.001 \%$ methylic alcohol \\
& & $0.016 \% \mathrm{H}_{2} \mathrm{O}$, \\
& & $0.002 \%$ alcohol, \\
& & $0.003 \%$ oil, \\
Schweissen Technik & 99.8 & $0.2 \%$ Alkane, $0.0001 \% \mathrm{~S}$ \\
Matheson & 99.5 & $0.1 \% \mathrm{CO}_{2}, 0.1 \%$ methyl formate, \\
& & $0.3 \%$ alcohol \\
\hline
\end{tabular}


certainly contained in the $\mathrm{Al}$ used for the tube, and it was found also on the O-rings. The $F$ certainly comes from the fluorocarbons, especially from the O-rings (Viton which, as we have learned since, is attacked by ethers). More difficult to establish is the origin of $\mathrm{Cl}$. It may simply have come from the insulation of the HV wire (PVC). However, $\mathrm{Cl}$ is also contained in Freon TF, perhaps used to clean the wire. Since there are known instances of contamination of DME by Freon, we have asked three manufacturers to give the exact composition of their gas. In table 2 we have collected these results. We have been assured that this analysis refers to the gas coming out of the bottle, rather than straight from the production process. The presence of methyl alcohol, and even water of $S$, is not surprising and indicates that all three manufacturers use the same process of dehydration of alcohol. The other impurities indicate that the bottles are used for liquefied gases other than DME, principally saturated hydrocarbons. Anyway all manufacturers have excluded the presence of Freons. An analysis of one of the three gases, done at CERN with a gas chromatograph, has shown the presence of contaminant hydrocarbons as stated by the manufacturers, but has not revealed the presence of Freons. We should add that the sensitivity of the instrument was only $0.01 \%$.

We have redone the ageing test using Kalrez (DuPont) O-rings (guaranteed resistant to ethers), using $\mathrm{Cu}$ tubing, a HV wire insulated with Teflon and the best possible conditions of cleanliness. After collecting $1 \mathrm{C} / \mathrm{cm}$, we have had neither a change in the current drawn by the wire, nor in its gain. Deposits were found on the entire length of the wire (final diameter: $40 \mu \mathrm{m}$ ); only $\mathrm{Si}$ was detected in them. It is a well-known fact that finding all sources of impurities is a long and complicated affair [6].

In addition, we have made tests to check the resistance to DME of some common materials used in chambers. We have tested epoxy, Mylar, Teflon, scintillator (NE 110), Vetronite (G10), Stesalit, Plexiglas, PVC, and carbon-fibre composite. The method was to keep the specimen for a month in the gas, weighing it and looking at the state of the surface before and after with the electron microscope. The weight of all specimens had gone up at the end of the test: it probably simply means that they had all absorbed some DME. Signs of damage were found on the surface of the Plexiglas and on the Stesalit, where the bonding agent between the fibres seemed to have been dissolved. The scintillator was visibly milky and its surface scaled. All the other specimens seemed in good condition.

\section{Conclusions}

1) Dimethyl ether is accurate. Tracks in the gain gap can be reconstructed with a good spatial resolution, especially optimizing $V_{\mathrm{f}}$ and $V_{\mathrm{s}}$. Systematic effects can be corrected for.

2) New measurements are needed to establish the value of primary ionization. We have, in fact, tried to simulate, with the Monte Carlo code, our experimental results. However, the calculated results are very sensitive to the number of primary ions.

3) Dimethyl ether does not grow whiskers. We have shown that a careful selection of the material is necessary in order to avoid or reduce deposits on the wire.

\section{Acknowledgements}

We would like to express our appreciation to the technical services of the INFN (Bologna) for designing the chamber (G. Laurenti and C. Alvisi) and to the technicians who built the pressure vessel. Our thanks also go to $\mathrm{H}$. Bienkowski for building the drift cell and helping to solve many other problems. The help of the group of G. Muratori, and especially of J.C. Bertrand, L. Bassi and R. Harfield, is also gratefully acknowledged. J. Va'vra (SLAC) has been so kind as to lend us his program, and A. Bassi adapted it to our VAX $11 / 780$ in Bologna. We also wish to thank G. Jesse for carrying out the gas chromatography, J. Adam for doing the X-ray fluorescence tests and C. Nuttall for advice on our resistance tests. Finally, we would like to express our gratitude to F. Villa (SLAC), without whom this work would not have been possible.

\section{References}

[1] F. Villa, Nucl. Instr. and Meth. 217 (1983) 273.

[2] M. Basile et al., Nucl. Instr. and Meth. A239 (1985) 497.

[3] ZEUS Collaboration, The ZEUS Detector, Technical Proposal, DESY-HERA (March 1986) p. 8-2.

[4] R. Desalvo, Nucl. Instr. and Meth. A235 (1985) 509.

[5] T.L. Cottrell et al., Trans. Faraday Soc. 61 (1965) 1585.

[6] J. Adam, Nucl. Instr. and Meth. 217 (1983) 291. 